${ }^{2}$ Sensor Physics Branch, NOAA/NESDIS/ORA, Camps Spring, Maryland, USA

${ }^{3}$ Department of Atmospheric Sciences, National Taiwan University, Taipei, Taiwan

${ }^{4}$ Naval Research Laboratory, Monterey, California, USA

\title{
Reanalysis of western Pacific typhoons in 2004 with multi-satellite observations
}

\author{
X. Zhang ${ }^{1}$, T. $\mathbf{L i}^{1}$, F. Weng ${ }^{2}$, C.-C. $\mathbf{W u}^{3}$, and L. $\mathbf{X u}{ }^{4}$ \\ With 8 Figures
}

Received July 11, 2006; accepted September 11, 2006

Published online: March 14, 2007 (C) Springer-Verlag 2007

\begin{abstract}
Summary
A pilot tropical cyclone reanalysis project was conducted to construct a reliable, high temporal and spatial resolution tropical cyclone dataset for selected western Pacific typhoons in summer 2004, with the application of the latest satellite observations and a 4-dimensional variational data assimilation method. Primary data used for the reanalysis include SSM/I rain rate, GOES-retrieved upper-level wind, QuikSCAT surface wind, Aqua AIRS/AMSU retrieved temperature and moisture profiles, and JTWC best track data. A regular reanalysis procedure was established and up to 12 western Pacific typhoons have been reanalyzed. The reanalysis period covers the entire life cycle of a tropical cyclone, from a few days prior to its genesis to its final decay stage. A preliminary analysis shows that the reanalysis product significantly improves typhoon intensity, structure, and track, compared to the NCEP operational final analysis. The validation of the TC structure against independent observations shows that the reanalysis reproduces well the asymmetric characteristics of TC rain bands and cloud bands. A further modeling experiment with an initial condition from the reanalysis product reveals a significant improvement in typhoon intensity forecast compared to a parallel experiment with an initial condition from the NCEP final analysis, which provides a further indication of quality of the tropical cyclone reanalysis. The reanalysis product and the raw observational data will soon be posted on the data server of the IPRC Asia-Pacific Data-Research Center (http://apdrc.soest.hawaii.edu/) for public use.
\end{abstract}

\section{Introduction}

Tropical cyclone (TC) is an extreme weather system that may cause devastating floods and considerable economic and human losses. While TC track forecasts have improved significantly and steadily over the last few decades, progress in storm intensity forecasting has been very slow (DeMaria, 2005). The lack of skill in the intensity forecasts may be attributed to several factors, such as insufficient horizontal and vertical resolution, inadequate physical parameterizations, and the absence of full coupling with the ocean in the current prediction models, but most importantly, the lack of knowledge about initial 3-dimensional TC structures due to insufficient observations over the ocean.

As demonstrated by many previous studies, the key factors controlling TC intensity are the inner core dynamics and interactions with the environmental flow. To foster the understanding of fundamental dynamics of TC and the interaction with the environmental flow, a comprehensive high-resolution dataset that covers the entire TC life cycle is needed. Up to now, only some coarse datasets (e.g., NCAR/NCEP or ECMWF reanalysis) were available, and their spatial and 
temporal resolutions are far from the detail we require to describe and analyze the inner-core TC structure, and the interactions among symmetric and asymmetric components and higher and lower wave-number perturbations. Aircraft radar and dropsondes may provide fine-scale TC motions and thermodynamic patterns, but the coverage is limited in both time (usually every 24 hours or so) and space (usually a few legs at one or two special levels).

Efforts have been made to improve TC structures using satellite products. For example, studies showed that the assimilation of multi-satellite remote-sensing data, such as surface vector winds from scatterometer data (Leidner et al, 2003) and satellite-retrieved tropospheric temperature profiles from the microwave data (Chen et al, 2004), has a positive impact on TC track and intensity simulation/forecast through improved model initial conditions. Zhang et al (2006) assimilated QuikSCAT surface wind data, GOESretrieved wind, and Aqua MODIS sounding data to investigate hurricane Lili (2002)'s rapid weakening. Their study suggested that assimilating QuikSCAT data and GOES-derived upper-level winds improves the analyzed outer-core winds and the inner-core low-level temperature and moisture fields significantly, while assimilating Aqua MODIS sounding data improves the outercore thermodynamic features and shows an impact on the model intensity prediction. Zhao et al (2005) showed the effect of a 4-dimensional variational (4DVAR) data assimilation scheme in assimilating irregularly distributed (in both space and time) observations such as AMS U-A retrieved temperature and wind fields, as well as the minimal sea-level pressure (MSLP) information. With a 72-hour simulation of a landfall typhoon, they concluded that both the satellite data and the MSLP information could improve the typhoon track forecast, especially for the recurving of the track and landing point.

While the satellite applications above were mainly for TC case studies, there is a need to develop a general strategy to best utilize the remote-sensing products and advanced data assimilation schemes. Motivated by this emerging need, we conducted a pilot project to construct a comprehensive, high-resolution reanalysis dataset for summer 2004 western Pacific typhoons (hereafter, referred to as TC-RA). We intended to take advantage of a variety of satellite products, such as QuikSCAT measured surface winds, GOES-8 cloud- and water-vapor-drifted upperlevel winds, and SSM/I rainfall rates. In particularly, the successful lunching of the second Earth Observing System polar orbiting platform satellite Aqua with its Atmospheric Infrared Sounder (AIRS) and the Advanced Microwave Sounding Unit (AMSU), in May 2002, provide us an unprecedented opportunity to obtain fine-resolution (about $5 \mathrm{~km}$ ) 3D atmospheric moisture and temperature fields with continuous coverage in time. These sensors constitute an innovative atmospheric sounding group of visible, infrared, and microwave detectors, and some of them can penetrate through deep convective clouds and provide high-resolution multi-channel radiance and moisture data. They provide new and improved temperature measurements with an accuracy of $1 \mathrm{~K}$ in layers of $1 \mathrm{~km}$ and humidity measurements with an accuracy of $20 \%$ in layers of $2 \mathrm{~km}$ in the troposphere. Since there are limited conventional observations over the open ocean where TCs occur and evolve, an effective use of remote-sensing data from satellite is crucial for improving TC initialization and prediction. In this study, we intend to utilize the aforementioned satellite products and a 4DVAR data assimilation approach to construct a continuous-coverage, high-resolution TC dataset.

Because our computer and data resources are limited, we selected 12 typhoons that occurred over the western Pacific region from May to October 2004 for this reanalysis (see Table 1). The primary data input into the data assimilation system includes time series of MSLP information from the best track, Aqua-measured moisture and temperature profiles, QuikSCAT surface wind fields, GOES-retrieved cloud-drifted winds, and $\mathrm{SSM} / \mathrm{I}$ rainfall rates. Our strategy is to combine these observations with model dynamics in order to derive dynamically balanced TC wind, pressure, moisture, and temperature fields during the entire TC life cycle.

The paper is organized as follows. In Sect. 2, we introduce the mesoscale model employed and its adjoint model, variational data assimilation formalism, and the model configuration. The usage of the satellite and other observational data, as well as the justification of their usefulness in TC initialization, are summarized in Sect. 3. 
Table 1. List of 12 typhoons selected in the TC-RA (from JTWC)

\begin{tabular}{lllll}
\hline TC & Name & Period & $\begin{array}{l}\text { Max sfc } \\
\text { wind (m/s) }\end{array}$ & $\begin{array}{l}\text { MSLP } \\
(\mathrm{hPa})\end{array}$ \\
\hline WP 7 & Conson & 04 Jun-11 Jun & 42.5 & 958 \\
WP 9 & Diamu & 13 Jun-21 Jun & 75 & 885 \\
WP10 & Mindulle & 22 Jun-04 Jul & 62.5 & 916 \\
WP12 & Kompasu & 13 Jul-16 Jul & 22.5 & 991 \\
WP16 & Rananim & 07 Aug-12 Aug & 45 & 954 \\
WP18 & Megi & 13 Aug-19 Aug & 32.5 & 976 \\
WP19 & Chaba & 18 Aug-31 Aug & 77.5 & 879 \\
WP20 & Aere & 19 Aug-26 Aug & 32.5 & 976 \\
WP22 & Songda & 27 Aug-07 Sep & 65 & 910 \\
WP25 & Meari & 20 Sep-29 Sep & 60 & 922 \\
WP26 & Ma-on & 04 Oct-09 Oct & 70 & 898 \\
WP27 & Tokage & 12 Oct-20 Oct & 60 & 922 \\
\hline
\end{tabular}

The demonstration of the performance of the reanalysis product is discussed in Sect. 4. A conclusion and discussions are given in Sect. 5.

\section{Data assimilation system}

For TC initialization, there is a clear need for the assimilation scheme that can initialize the vortex with the correct position, intensity and structure (including perhaps its asymmetric components) and minimize spurious transient evolutions in the subsequent forecast. In-situ observations are typically too sparse to characterize TC mesoscale flows and thus data assimilation in the TC innercore region often relies on remotely sensed observations and the ability of a data-assimilation system to project the limited data onto TC dynamic structures. Since remotely sensed observations in many cases have a complicated and indirect relation to the model prognostic variables, the data assimilation algorithms applied must be capable of utilizing these diagnostic variables such as $\mathrm{SSM} / \mathrm{I}$ rain rates.

Advanced assimilation schemes such as the 4DVAR or ensemble Kalman filter (EnKF) have great potential to improve TC initial conditions. Different from 3-dimensional variational (3DVAR) method in which the influence of a single observation on the analysis is independent of the presence of the TC vortex, the advanced assimilation schemes such as the 4DVAR and EnKF schemes, in contrast, use dynamical information from the forecast and their analysis increments depend on the presence of the vortex. The latter schemes can effectively shift the location of the vortex given limited observations (Leidner et al, 2003; Chen and Snyder, 2006).

\subsection{The mesoscale model}

The Penn State University/National Center for Atmospheric Research (PSU/NCAR) mesoscale forecast model (MM5) was used in this research. The MM5 is a limited-area, non-hydrostatic model with multiple options for various physical parameterization schemes (Dudhia, 1993; Grell et al, 1995). The model employs a terrain-following $\sigma$ vertical coordinate, where $\sigma$ is defined as $\sigma=\left(p-p_{\text {top }}\right) /\left(p_{\text {sfc }}-p_{\text {top }}\right), p$ is pressure, and $p_{\text {sfc }}$ and $p_{\text {top }}$ are the pressure at the surface and model top, respectively. The model consisted of 30 vertical levels from surface to $100 \mathrm{hPa}$, the 29 half- $\sigma$ levels are $0.025,0.075,0.125,0.175,0.225$, $0.275,0.325,0.375,0.425,0.525,0.575,0.625$, $0.660,0.685,0.715,0.740,0.760,0.785,0.815$, $0.840,0.860,0.885,0.910,0.930,0.950,0.965$, $0.975,0.985,0.995$. In the horizontal direction, the model has 2 nested typhoon center-followed movable domains. The coarser mesh has $72 \times 72$ grids with $90-\mathrm{km}$ resolution and the finer mesh has $100 \times 100$ grids with $30-\mathrm{km}$ resolution.

Physics parameterizations used for the mesoscale model include the Grell cumulus parameterization and a simple ice microphysics scheme (Dudhia, 1993) for the coarser domain, the KainFritsch cumulus model (Kain and Fritsch, 1990; 1993) and the Reisner graupel (Reisner et al, 1998) microphysics scheme for the finer domain, the Blackadar high-resolution planetary boundary layer (PBL) parameterization scheme (Zhang and Anthes, 1982), and a cloud atmospheric radiation scheme (Dudhia, 1993) for all domains. The SST data from the NCEP global analysis are updated daily during the assimilation, when the surface heat and moisture fluxes are calculated. A radiative boundary condition is applied in the upper boundary to deal with the vertically propagating waves. The reader may refer to Dudhia (1993) and Grell et al (1995) for more detailed descriptions of the model dynamics and physics.

\subsection{The adjoint model}

We employ the MM5-4DVAR data assimilation system developed by AFWA (Ruggiero et al, 


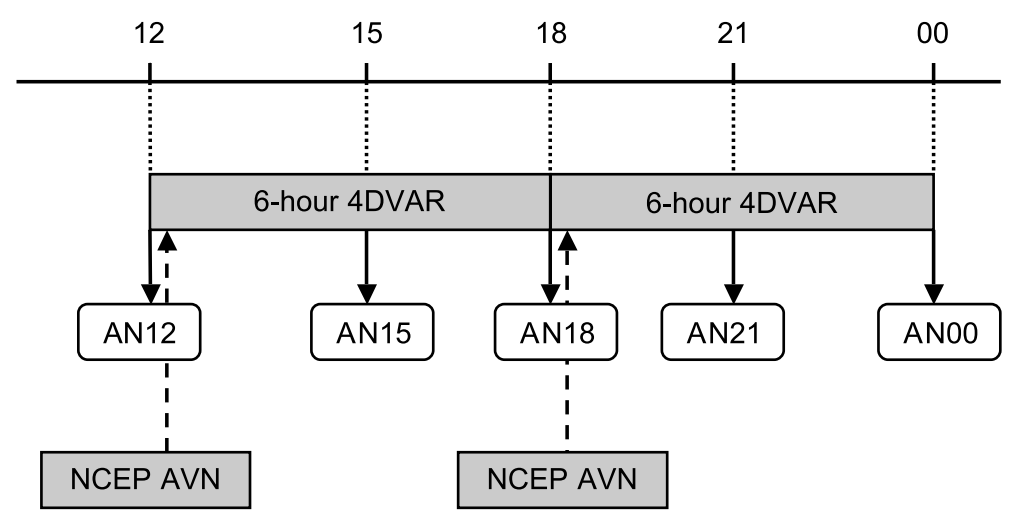

Fig. 1. Data assimilation cycling for the 4DVAR

2006). This MM5-4DVAR system has been parallelized based on the same method applied to the MM5 model and can be run on SGI Altix, SGI Origin series and IBM SP series effectively. Before using this system, both gradient and adjoint checks were conducted to ensure its correctness. Currently the 4DVAR system contains physical parameterization schemes such as stable precipitation scheme, Kuo convective cumulus parameterization scheme (Kuo, 1974), grid-scale precipitation and MRF PBL scheme (Troen and Mahrt, 1986). The minimization algorithm used here is the limited-memory quasi-Newton method developed by Liu and Nocedal (1989).

The 4DVAR system tries to minimize the following general cost function:

$J\left(\mathbf{x}_{0}\right)=J_{b}\left(\mathbf{x}_{0}\right)+\sum_{o=1, m} J_{o}\left(\mathbf{x}_{0}\right)+J_{c}\left(\mathbf{x}_{0}\right)$,

where $\mathbf{x}_{0}$ is the analysis variable, which includes wind $(\mathbf{u}, \mathbf{v})$, temperature $(\mathbf{T})$, specific humidity $(\mathbf{q})$, and pressure perturbation $\left(\mathbf{p}^{\prime}\right)$ from a constant reference state, and subscript " 0 " denotes the initial state; $J_{b}$ is a simple background term measuring the distance between the model state and the MM5 analysis field (based on the global NCEP analysis), which is expressed as

$J_{b}\left(\mathbf{x}_{0}\right)=\frac{1}{2}\left[\mathbf{x}_{0}-\mathbf{x}_{b}\right]^{T} \mathbf{B}^{-1}\left[\mathbf{x}_{0}-\mathbf{x}_{b}\right]$,

where $\mathbf{B}$ is the background error covariance and a diagonal matrix proportional to the inverse of the root-mean-square difference between the 12-h forecast and the MM5 analysis fields, and $J_{o}$ is the sum of contributions from each of five types of observational data (i.e., TC best track, QuikSCAT surface wind, GOES retrieved wind, SSM/I rain rate, and Aqua retrieved temperature and water vapor) described in Sect. 3. The subscript " $o$ " here denotes the type of the observation and $m$ is the total number of available observation types. $J_{c}$ is a weak constraint term for reducing highfrequency noises and enhancing the balance of atmospheric states, and will be described in subsection d.

\subsection{Analysis cycling}

The reanalysis cycling was configured with 4 cycles per day of 6-hour 4DVAR, Fig. 1 gives an overview of the data flow through the data assimilation system. The 1800 UTC assimilation uses observations from the time window 1201-1800 UTC. Its first guess is interpolated from 1200 UTC NCEP analysis. It generates two sets of analyzed fields, at 1500 and 1800 UTC. The 0000 UTC analysis uses observations from the time window 1801-0000 UTC. Its first guess is interpolated from the 1800 UTC NCEP analysis. It generates analyzed fields at 2100 and 0000 UTC.

\subsection{Noise control}

The 4DVAR is a method that seeks a model trajectory that best fits a set of observations in a given time window. The so-generated analysis is consistent with the model dynamics. Nevertheless, in the case of insufficient observations in either the space or time dimension, a key issue is whether the solution is unique or not (Zou et al, 1992). Note that the "optimization" from the traditional 4DVAR does not necessarily provide the best, physically reasonable atmospheric or ocean state because it only concerns the minimization of 
a cost function under constraints imposed. Wee and Kuo (2004) pointed out that the traditional 4DVAR had a tendency to excite high-frequency waves to fit observations so that the cost function can be rapidly reduced. This is because these high-frequency modes contain less energy compared to slow-varying modes, and may result in unrealistic atmospheric states.

Lynch and Huang (1992) proposed a digital filter initialization (DFI) method that can suppress high-frequency oscillations effectively. The DFI is simple in both its basic concept and implementation compared to other methods. It has been implemented into several operational 4DVAR data assimilation models as a penalty term (JcDFI), such as in ECMWF, HIRLAM (Huang and Lynch, 1993) and Météo-France (Gauthier and Thépaut, 2001). The performance of JcDFI has been demonstrated in the study of blizzard (Zupanski and Zupanski, 2002), rainfall data assimilation (Peng and Zou, 2002), and Antarctic cyclogenesis (Wee and Kuo, 2004).

The $J_{c}$ in Eq. (1) is a JcDFI penalty term that measures the distance between a filtered state and the corresponding unfiltered state, and thus it reflects the amount of undesirable high-frequency gravity waves during the data assimilation.

$J_{c}\left(\mathbf{x}_{0}\right)=\frac{1}{2}\left(\mathbf{x}_{3 h}-\overline{\mathbf{x}}_{3 h}\right)^{T} \mathbf{D}\left(\mathbf{x}_{3 h}-\overline{\mathbf{x}}_{3 h}\right)$,

where $\mathbf{D}$ is a diagonal matrix; $i$-th diagonal element of $\mathbf{D}$ can be expressed as $d_{i}=\left(\lambda / \bar{\sigma}_{b}^{2}\right)_{i}$, and $\lambda$ is the weighting factor for each variable type, and $\bar{\sigma}_{b}^{2}$ is the global variance of the background error; $\mathbf{x}_{3 h}$ is an unfiltered state defined at the middle of the 6-h time window, and $\overline{\mathbf{x}}_{3 h}$ is the corresponding filtered state. Detailed description of the implementation of digital filter in MM5 4DVAR system can be found in the paper of Wee and Kuo (2004).

A series of observing system simulation experiments (OSSEs) was conducted to assess the impact of the JcDFI on TC initialization (see Fig. 2). A three-dimensional wind field from a simulated TC with a MSLP of $976 \mathrm{hPa}$ is regarded as a "true" TC state (denoted as "True"), while a degraded TC with a MSLP of $1002 \mathrm{hPa}$ represents the first guess (denoted as "No_Data"). The penalty term of JcDFI was evaluated in two parallel experiments (with and without the
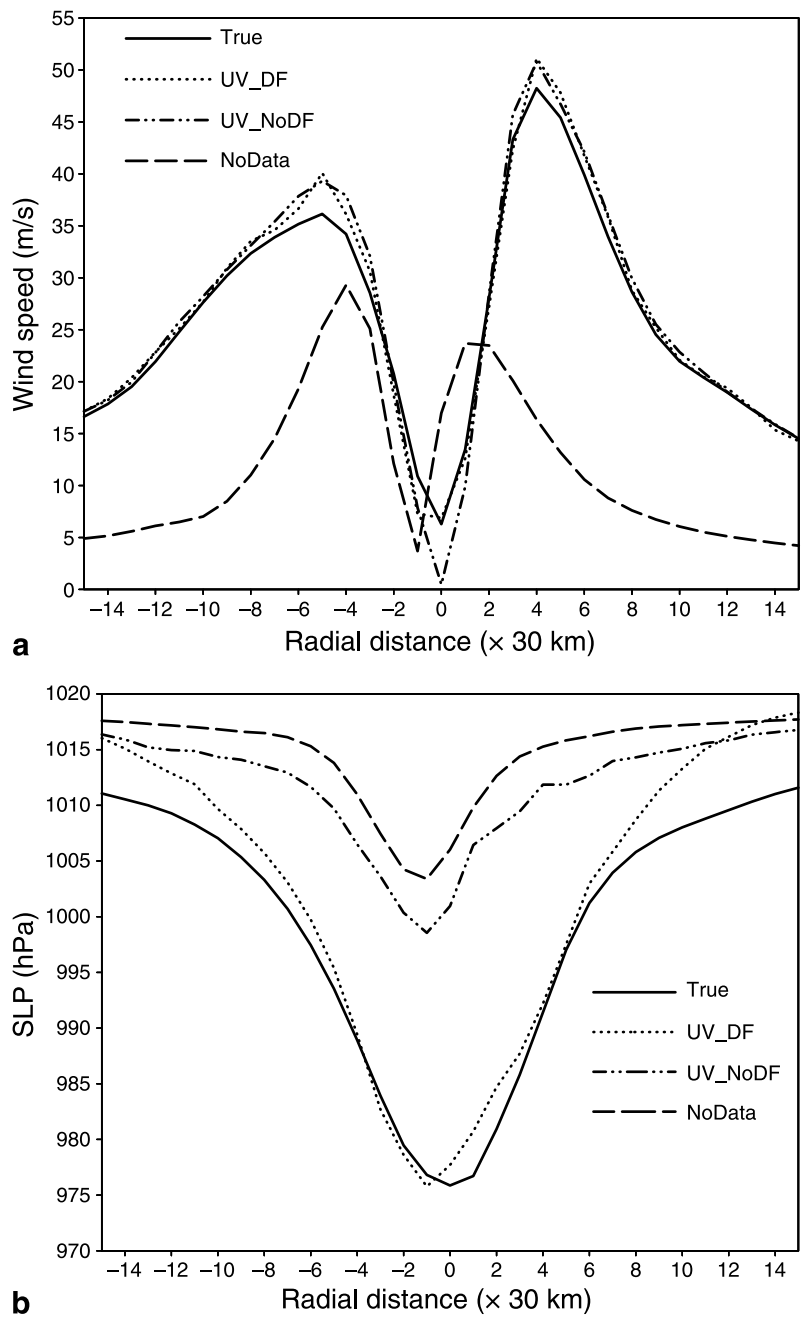

Fig. 2. West-east cross section of (a) the low-level wind speed profile, and (b) the SLP profile along the TC center for the first guess field (NoData), the true state (True), the assimilation of the 3D winds with the DFI (UV_DF), and the assimilation of the winds without DFI (UV_NoDF) at $0 \mathrm{~h}$

JcDFI term) to investigate its impact on the performance of the TC structure reconstruction, given the "true" 3D wind field. Figure 2 shows the east-west cross section (through the TC center) of low-level wind speed and SLP profiles from the two experiments with JcDFI (UV_DF) and without JcDFI (UV_NoDF). As expected, the assimilated winds fields in both experiments match the "true" state closely (Fig. 2a). However, a significant difference appears in the SLP profiles (Fig. 2b). The analyzed MSLP in UV_NoDF is $998 \mathrm{hPa}$ while it reaches $976 \mathrm{hPa}$ in UV_DF. This result indicates that the weakly constrained 4DVAR with the JcDFI reproduces a balanced initial TC structure in both the mass and wind fields. 


\section{Observational data and cost functions}

\subsection{JTWC best track}

The JTWC maintains an archive of TC track data, commonly referred to as "best track". Each of the best track files contains TC center locations and intensities (i.e., the MSLP and the maximum 1-minute mean sustained 10-meter wind speed) at a six-hour interval. The geographical domains of the archive are the western North Pacific (WP), North Indian Ocean (IO), and Southern Hemisphere (SH). A 4DVAR bogus data assimilation (BDA) scheme was developed (Zou and Xiao, 2000; Xiao et al, 2000; Park and Zou, 2004) based on the best track TC center. To make the BDA scheme work, it is necessary to specify a bogus vortex that represents the main features of the corresponding storm, such as its size and intensity, at the initial time of the forecast (Zou and Xiao, 2000; Pu and Braun, 2001). Either the Fujita (1952) formula or Holland (1980) formula is used to formulate an axisymmetric SLP pattern, with the specified central SLP, environmental pressure, radius of the maximum wind or SLP gradient, and the radius of the outermost closed isobar. Most of the parameters above can be determined with the best track data. However, direct information on the radius of the maximum SLP gradient is not available and thus it is specified arbitrarily. This BDA scheme usually produces a concentric, symmetric TC structure at the initial time.

To let the asymmetric TC structure evolve freely during the data assimilation while keeping the observed TC intensity, here we introduce a so-called point pressure (PP) scheme. In this scheme, we interpolate 6-hour best track data into 0.5-hour interval and assimilate the onepoint central MSLP sequentially every half hour over the 6-hour time window. It is expected that this scheme allow the development of asymmetric TC component in response to satellite-derived heating while TC position and intensity are close to the best track. To test this scheme, we again designed a set of OSSEs. With the "true" temperature and water vapor profiles known, we investigate the impact of the PP scheme on the TC initialization. Figure 3 shows that when only the temperature and water vapor are assimilated (Fig. 3a), limited improvement on TC intensity is

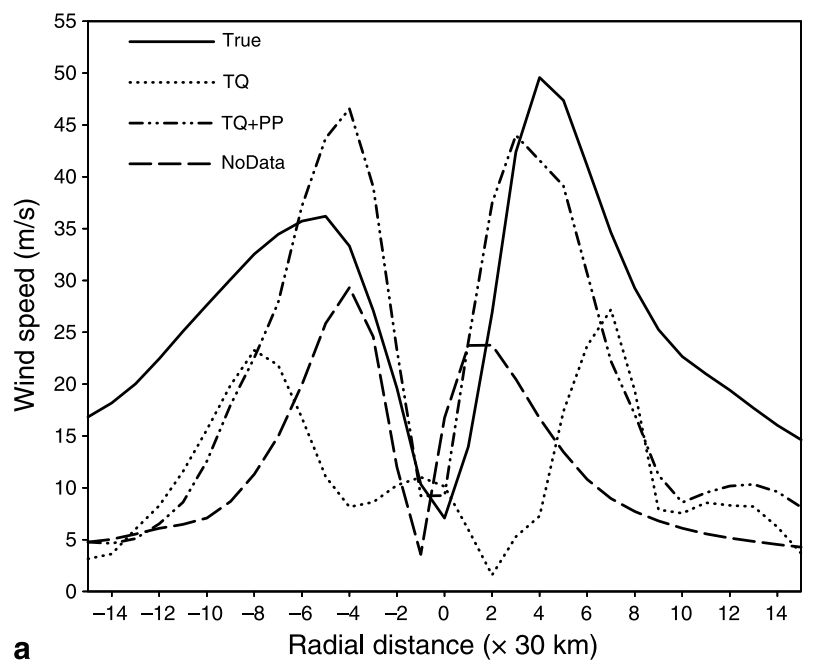

a

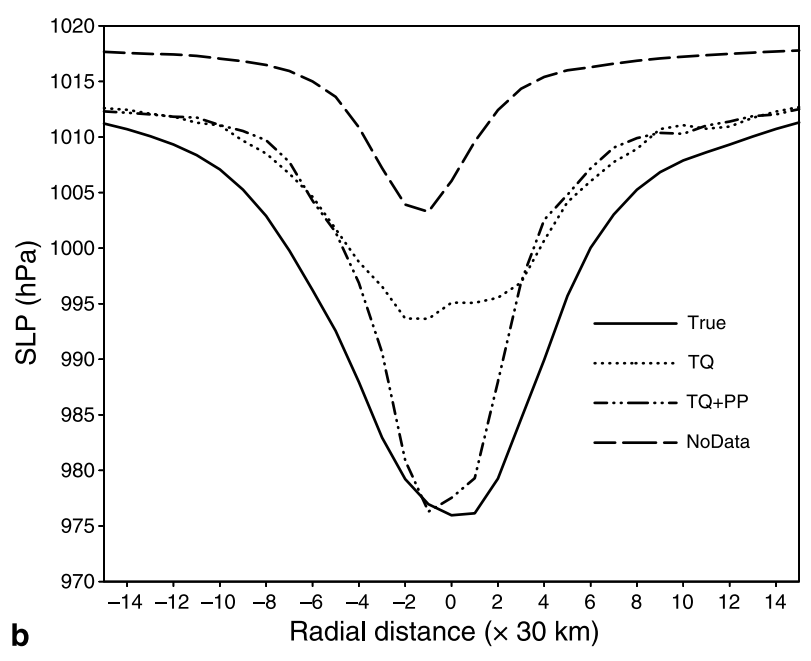

Fig. 3. West-east cross section of (a) the low-level wind speed profile, and (b) the SLP profile along the TC center for the first guess, the true state, assimilation with both temperature and water vapor given, and assimilation with temperature, water vapor and point pressure together

observed; on the other hand, when the PP scheme was included (together with the assimilated temperature and water vapor), both the SLP and wind profiles fit well the "observation". It has been shown that the assimilation of both the satelliteretrieved data and moving information of the typhoon center may improve TC track forecast (Zhao et al, 2005).

In this reanalysis effort, we use this PP scheme. Note that this pressure assimilation scheme is particularly desired when incorporating satellite observations such as GOES retrieved winds, QuikSCAT scatterometer winds, and SSM/I rain rates. Otherwise, TC bogus scheme will be employed. 
The observational cost function for the PP scheme may be defined as

$$
\begin{aligned}
J_{P P}\left[\mathbf{x}_{0}\right]= & \frac{1}{2} \sum_{i=0}^{N}\left\{\left[P P\left(t_{i}\right)-P P^{o b s}\left(t_{i}\right)\right]^{T}\right. \\
& \left.\times \mathbf{W}_{P P}\left[P P\left(t_{i}\right)-P P^{o b s}\left(t_{i}\right)\right]\right\},
\end{aligned}
$$

where $\operatorname{PP}\left(t_{i}\right)$ represents the model-predicted SLP at the location of the best track at time $t_{i}$ and $\mathrm{PP}^{\text {obs }}\left(t_{i}\right)$ is the observational MSLP at the same time; $N$ is total number of observations available; $\mathbf{W}_{\mathrm{PP}}$ is the weighting for MSLP and simply taken as $1 \mathrm{mb}^{-2}$.

\subsection{SSM/I rainfall data}

The SSM/I onboard the Defense Meteorological Satellite Program (DMSP) satellite is a sevenchannel, four-frequency, linear-polarized, passive microwave radiometric system that measures atmospheric, ocean, and terrain microwave brightness temperature at 19.35, 22.235, 37.0, and 85.5 GHz. All frequencies are received in dual polarization only. The active portion of the conical scan covers a swath of about $1400 \mathrm{~km}$, and the geometric resolution of processed brightness temperatures is $25 \mathrm{~km}$ (except $85.5 \mathrm{GHz}$ ). For the 2004 typhoon season, observations from F13, F14 and F15 are available. Several atmospheric parameters can be retrieved from SSM/I observations (Hollinger, 1991). Among them the total rain rate (RR) and precipitable water (PW) are of primary interest for numerical weather prediction. The impact of SSM/I-derived RR and PW, as additional sources of moisture information on numerical forecasting of tropical circulations (Ledvina and Pfaendtner, 1995; Tsuyuki, 1997) and tropical cyclones (Shi et al, 1996; Peng and Chang, 1996; Karyampudi et al, 1998), has been extensively investigated.

The assimilation of $\mathrm{RR}$ into the mesoscale model is not as straightforward as the conventional prognostic variables such as wind, temperature and specific humidity since RR is an indirect, diagnostic 2D variable. Previous studies such as Zupanski and Mesinger (1995), Zou and Kuo (1996), Xiao et al (2000), and Zhang et al (2003) successfully assimilated rainfall observations using a 4DVAR approach. Their studies demonstrate a positive impact on precipitation forecast. Pu et al $(2002 ; 2004)$ studied the impact of the R R data derived from the Tropical Rainfall Measuring Mission (TRMM) Microwave Imager on hurricane Bonnie (1998) and supertyphoon Paka (1997) with the MM5 4DVAR. Their results showed the improvement of the rainfall assimilation in TC structure and intensity forecast, in particular, when the rainfall data are incorporated with other types of data such as winds and a bogus vortex.

The cost function for the RR is defined as

$$
\begin{aligned}
J_{R R}\left[\mathbf{x}_{0}\right]= & \frac{1}{2} \sum_{i=0}^{N}\left\{\left[\mathbf{R} \mathbf{R}\left(t_{i}\right)-\mathbf{R R}^{o b s}\left(t_{i}\right)\right]^{T}\right. \\
& \left.\times \mathbf{W}_{R R}\left[\mathbf{R} \mathbf{R}\left(t_{i}\right)-\mathbf{R R}^{o b s}\left(t_{i}\right)\right]\right\},
\end{aligned}
$$

where $\mathbf{R R}\left(t_{i}\right)$ represents the model-predicted convective and stratiform rainfall at time $t_{i}$ and $\mathbf{R R}^{\text {obs }}\left(t_{i}\right)$ is the SSM/I measured RR at the same time; $N$ is total number of observations available; $\mathbf{W}_{R R}$ is the weighting for RR and simply taken as $1 \mathrm{~mm}^{-2} / \mathrm{h}^{-2}$. To ensure the quality of $\mathrm{RR}$ data into the data assimilation system, we removed any data contaminated by sea ice, land and heavy rain.

\subsection{GOES-retrieved wind}

High-density (resolution) wind vector fields may be derived from cloud and water vapor motions by taking advantage of the improved radiometric observations from GMS-5 and GOES-9 satellites (Velden et al, 1998). The radiance measured from GMS-5 or GOES-9 can be converted into imagery, and selected spatial discontinuities (targets) are then tracked over a 30-min interval to obtain motion vectors. The satellite-retrieved wind data are then derived through automated tracking algorithms applied to water vapor (WV) fields and visible (VIS) and infrared (IR) imagery (Nieman et al, 1997; Velden et al, 1997; 1998). While these wind vectors represent layer-mean motions in most cases, their heights are determined by a combination of radiometric signals and other multivariate information. The detailed information about the targeting, tracking, height estimation, and quality control procedures may be found in Velden et al (1997; 1998).

The satellite wind data provide good coverage of large-scale synoptic patterns and sometimes a certain mesoscale flow patterns. Several studies (e.g., Le Marshall et al, 1985; Mills et al, 1986; 
Velden et al, 1992; 1998; Soden et al, 2001) have shown that satellite wind data have a positive impact on tropical forecasting. Higher density cloud- drift winds have been shown to improve the accuracy of cyclone track forecasting in the northwest Pacific with a barotropic variational assimilation scheme (Bennett et al, 1993). Bennett et al (1996) further showed that higher (spatial) density winds could improve hurricane track forecasts with a 4DVAR assimilation approach. The studies of Leslie et al (1998) and Velden et al (1998) provided strong evidence of the advantage of assimilating high-spatial and temporal resolution satellite-derived wind data on TC track forecast. Zou and Xiao (2000) showed that the initial hurricane vertical velocity structure is improved for hurricane Felix (1995) when the GOES-8 satellite wind was assimilated. Xiao et al (2002) assessed the impact of satellite-derived wind observations on the prediction of a mid-Pacific Ocean cyclone using the MM5 4DVAR system. They found that the assimilation of both GMS-5 and GOES-9 derived winds increases the cyclonic zonal wind shear and the cross-front temperature gradient.

In this study, the partial cost function that measures the discrepancy between the model-predicted and satellite-retrieved winds is defined as

$$
\begin{aligned}
J_{G O E S}\left[\mathbf{x}_{0}\right]= & \frac{1}{2} \sum_{t_{i}} \sum_{r_{l}}\left\{\left[\mathbf{H}_{l} \mathbf{u}-\mathbf{u}^{G O E S}\left(\mathbf{r}_{l}\right)\right]^{T}\right. \\
& \times \mathbf{W}_{u}\left[\mathbf{H}_{l} \mathbf{u}-\mathbf{u}^{G O E S}\left(\mathbf{r}_{l}\right)\right] \\
& +\left[\mathbf{H}_{l} \mathbf{v}-\mathbf{v}^{G O E S}\left(\mathbf{r}_{l}\right)\right]^{T} \\
& \left.\times \mathbf{W}_{v}\left[\mathbf{H}_{l} \mathbf{v}-\mathbf{v}^{G O E S}\left(\mathbf{r}_{l}\right)\right]\right\}
\end{aligned}
$$

where $\mathbf{r}_{l}$ is the vector of physical locations of the available satellite winds at time $\mathrm{t}_{i} ; \mathbf{H}_{l}$ is a linear interpolation scheme; and $\mathbf{W}_{u}$ and $\mathbf{W}_{v}$ are treated as constants and determined empirically. Here we assign $\mathbf{W}_{u}$ and $\mathbf{W}_{v}$ a value of $1 \mathrm{~m}^{-2} \mathrm{~s}^{2}$, which corresponds to a $1 \mathrm{~m} \mathrm{~s}^{-1}$ error for the satellitederived winds. Vectors $\mathbf{u}^{G O E S}$ and $\mathbf{v}^{G O E S}$ represent retrieved satellite zonal and meridional wind components, respectively. Two quality marks, Recursive Filter Flag (RFF) developed by Cooperative Institute for Meteorological Studies (CIMSS) (Hayden and Purser, 1995) and Quality Indicator (QI) developed by European Organization for the Exploitation of Meteorological
Satellites (EUMETSAT) (Holmlund, 1998), are currently used to estimate the GOES-retrieved wind quality. RFF mainly measures the certainty of the height assignment, while QI primarily addresses internal consistency during the retrieval process. Only higher quality vectors with the CIMSS RFF equal to or exceeding 50 and the EUMETSAT QI above 0.6 are passed to the data assimilation (Holmlund et al, 2001).

\subsection{Aqua AIRS/AMSU/HSB data}

AIRS, AMSU, and HSB constitute an innovative atmospheric sounding group of visible, infrared, and microwave sensors that provide measurements for temperature at an accuracy of $1{ }^{\circ} \mathrm{C}$ in layers $1 \mathrm{~km}$ thick and humidity with an accuracy of $20 \%$ in layers $2 \mathrm{~km}$ thick in the troposphere. The profile vertical resolution is 28 points total between $1000 \mathrm{hPa}$ and $0.02 \mathrm{hPa}$ and the horizontal resolution is $50 \mathrm{~km}$. The increased accuracy of the temperature and humidity profiles derived from AIRS/AMSU/HSB should enable improved forecasts from meteorological prediction models, and this represents an important component of satellite-based measurements designed to detect and quantify global climate change. Zhao et al (2005) studied the impact of three-dimensional AMSU-A-retrieved wind and temperature on typhoon forecasts with a MM5 4DVAR system. Their experiments showed that (1) the assimilation of the satellite-retrieved data was found to have a positive impact on the typhoon track forecast, (2) the assimilation of both the satelliteretrieved data and moving information of the typhoon center dramatically improved the track forecast and simulated the direction change and landfall very well, and (3) the assimilation of both the satellite-retrieved data and the bogus surface wind improved the intensity and track forecasts more significantly than the assimilation of the bogus surface wind only. Recently, Zhang et al (2006) included Terra-retrieved temperature and water vapor, QuikSCAT wind, GOES wind, and TMI water vapor into their MM5 4DVAR experiment for hurricane Lili (2002). Their study has yielded important insights into hurricane Lili's unexpected loss of punch in 2002. They found that the weakening was partially caused by low-level drier air moving into the west side of the storm, which was not detected at the time. 
The observational cost function for Aquaretrieved temperature and water vapor profiles may be defined as

$$
\begin{aligned}
J_{\text {Aqua }}\left[\mathbf{x}_{0}\right]= & \frac{1}{2} \sum_{t_{i}} \sum_{r_{l}}\left\{\left[\mathbf{H}_{l} \mathbf{T}-\mathbf{T}^{\text {Aqua }}\left(\mathbf{r}_{l}\right)\right]^{T}\right. \\
& \times \mathbf{W}_{T}\left[\mathbf{H}_{l} \mathbf{T}-\mathbf{T}^{\text {Aqua }}\left(\mathbf{r}_{l}\right)\right] \\
& +\left[\mathbf{H}_{l} \mathbf{q}-\mathbf{q}^{\text {Aqua }}\left(\mathbf{r}_{l}\right)\right]^{T} \\
& \left.\times \mathbf{W}_{q}\left[\mathbf{H}_{l} \mathbf{q}-\mathbf{q}^{\text {Aqua }}\left(\mathbf{r}_{l}\right)\right]\right\}
\end{aligned}
$$

where $\mathbf{r}_{l}$ is the vector of physical locations of the available observations at time $t_{i}$; vectors $\mathbf{T}^{\text {Aqua }}$ and $\mathbf{q}^{\text {Aqua }}$ represent Aqua-retrieved temperature and water vapor profile, respectively; $\mathbf{H}_{l}$ is a linear interpolation scheme; $\mathbf{T}$ is the model-predicted temperature profile and $\mathbf{q}$ is the modelpredicted water vapor profile at location $\mathbf{r}_{l}$ and at time $\mathrm{t}_{i} . \mathbf{W}_{T}$ and $\mathbf{W}_{q}$ are treated as constants and determined empirically. We assign $\mathbf{W}_{T}$ and $\mathbf{W}_{q}$ values of $1 \mathrm{~K}^{-2}$ and $1 \mathrm{~g}^{-2} / \mathrm{kg}^{-2}$, respectively.

\subsection{QuikSCAT surface wind}

The NAS A Quick Scatterometer (QuikSC AT) satellite carries the SeaWinds instrument, launched on 19 June 1999. It is the first satellite-borne scanning radar scatterometer designed to provide accurate ocean surface winds in all conditions (except for moderate to heavy rain) with an $1800-\mathrm{km}$-wide measurement swath on the earth's surface. The capability of measuring both wind speed and direction is the major characteristic of the scatterometer. The idea of remote sensing of ocean surface winds was based on the assumption that the roughness of the ocean surface is in equilibrium with the local wind stress. QuikSCAT provides twice-daily coverage over a given geographic region. Wind retrievals have been done on a $25 \mathrm{~km} \times 25 \mathrm{~km}$ grid. The quality of the scatterometer vector wind fields has been rigorously validated (e.g., Freilich and Dunbar, 1999; Ebuchi et al, 2002; Pickett et al, 2003) and applied to various scientific applications (Liu, 2002). The root-mean-square differences between ship measurements and QuikSCAT estimations are about $1 \mathrm{~m} \mathrm{~s}^{-1}$ in speed and $15^{\circ}$ in direction (Bourassa et al, 2003). However, optimal use of the QuikSCAT winds requires proper quality control based on knowledge of the special characteristics of these data.

A particular concern for the scatterometer wind data is that multiple winds or ambiguities are retrieved at each location (Price, 1976). The 4DVAR system has the potential to remove the ambiguity, because it uses scatterometer data at time of each observation, thereby avoiding errors caused by assuming that the data are collected at standard weather forecast times. Scatterometer data were shown to greatly improve both TC intensity and position errors in 1995 Atlantic hurricane season with the ECMWF 4DVAR system (Isaksen and Stoffelen, 2000). Case studies of hurricane Lili and typhoons Yates and Zane (occurring in 1996) showed similar positive impacts on intensity and track forecasts (Leidner et al, 2003).

The observational term in cost function that measures the distance between the model-predicted and QuikSCAT measured surface winds is defined as

$$
\begin{aligned}
J_{Q S C A T}\left[\mathbf{x}_{0}\right]= & \frac{1}{2} \sum_{t_{i}} \sum_{r_{l}}\left\{\left[\mathbf{H}_{Q S C A T} \mathbf{u}-\mathbf{u}^{Q S C A T}\left(\mathbf{r}_{l}\right)\right]^{T}\right. \\
& \times \mathbf{W}_{u}\left[\mathbf{H}_{Q S C A T} \mathbf{u}-\mathbf{u}^{Q S C A T}\left(\mathbf{r}_{l}\right)\right] \\
& +\left[\mathbf{H}_{Q S C A T} \mathbf{v}-\mathbf{v}^{Q S C A T}\left(\mathbf{r}_{l}\right)\right]^{T} \\
& \left.\times \mathbf{W}_{v}\left[\mathbf{H}_{Q S C A T} \mathbf{v}-\mathbf{v}^{Q S C A T}\left(\mathbf{r}_{l}\right)\right]\right\}
\end{aligned}
$$

where $\mathbf{r}_{l}$ is the vector of physical locations of the available sea surface winds at $\mathrm{t}_{i} ; \mathbf{H}_{Q S C A T}$ is a linear interpolation scheme; and $\mathbf{W}_{u}$ and $\mathbf{W}_{v}$ are treated as constants and determined empirically. We assign $\mathbf{W}_{u}$ and $\mathbf{W}_{v}$ a value of $1 \mathrm{~m}^{-2} \mathrm{~s}^{2}$, which corresponds to a $1 \mathrm{~m} \mathrm{~s}^{-1}$ error that is assumed in the satellite-derived winds. Vector $\mathbf{u}^{Q S C A T}$ and $\mathbf{v}^{Q S C A T}$ represent sea surface zonal and meridional wind components, respectively. It is well known that rain may affect the accuracy of scatterometers, resulting in erroneous cross track vectors and/or unrealistically high speeds. We discard the contaminated data in quality control based on the scatterometer-derived rainflag.

\section{Reanalysis results}

The goal of this TC reanalysis effort is to construct a three-dimensional high-resolution dataset that provides a continuous record of TC life cycle 
from May 2004 to October 2004 in the western Pacific. Up to the present, 12 high-impact typhoons whose life cycle is longer than two days and whose MSLP is lower than $995 \mathrm{hPa}$ have been reanalyzed. Several aspects of the quality of the TC-RA product are discussed below, by validating the reanalyzed TC intensity, track, and structure, and by conducting a hindcast experiment with the initial condition from the reanalysis field. The validation is against JTWC best track data for intensity and location and against independent satellite (TMI) observation for structure.

\subsection{Track and intensity}

The TC-RA product is verified against the JTWC best-track post analysis of all available track and intensity data. Here the TC intensity is defined as the MSLP and the TC position is defined as the location of the MSLP. For a very weak TC, the cyclone center of the surface wind field will
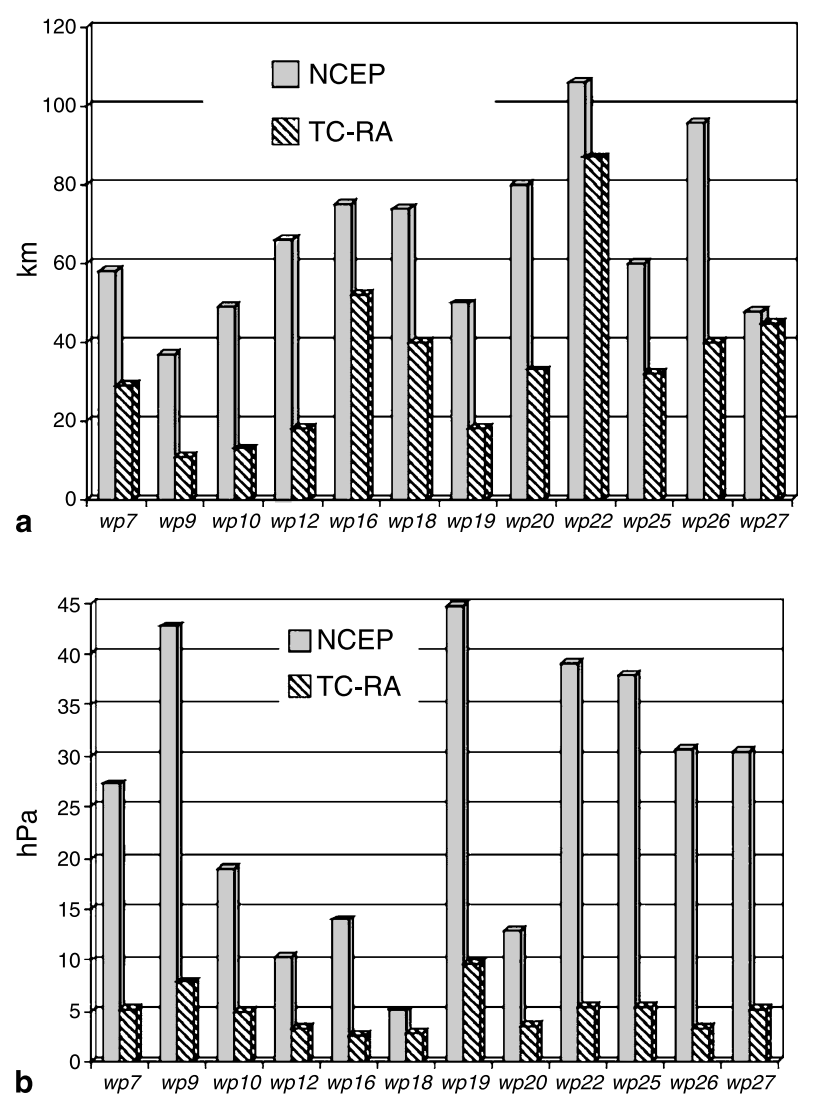

Fig. 4. The comparison of (a) the track bias (unit: km), and (b) the intensity bias (unit: $\mathrm{hPa}$ ) between the TC-RA production and the NCEP global analysis be determined as the TC position. Intensity errors are determined as the difference between the reanalyzed and the best-track MSLP at the same time and are presented as the average of the absolute magnitude of the error. Track analysis errors are determined as the great circle distance between the TC-RA position and the best track position at the same time.

The averaged intensity and track errors of the TC-R A product are presented in Fig. 4. The analysis biases of the NCEP global final analysis are also plotted for comparison. In Fig. 4a, the average track error bias of the NCEP global analysis for the 12 TCs is about $60 \mathrm{~km}$, whereas the TC-RA product reduces the track bias to around $30 \mathrm{~km}$. The most significant improvement is the TC intensity estimation (see Fig. 4b). The averaged error for the NCEP global analysis is about $30 \mathrm{hPa}$, while the TC-RA product is about 5-10 hPa.

Figure 5 shows the evolution of minimum central pressures for western Pacific typhoons
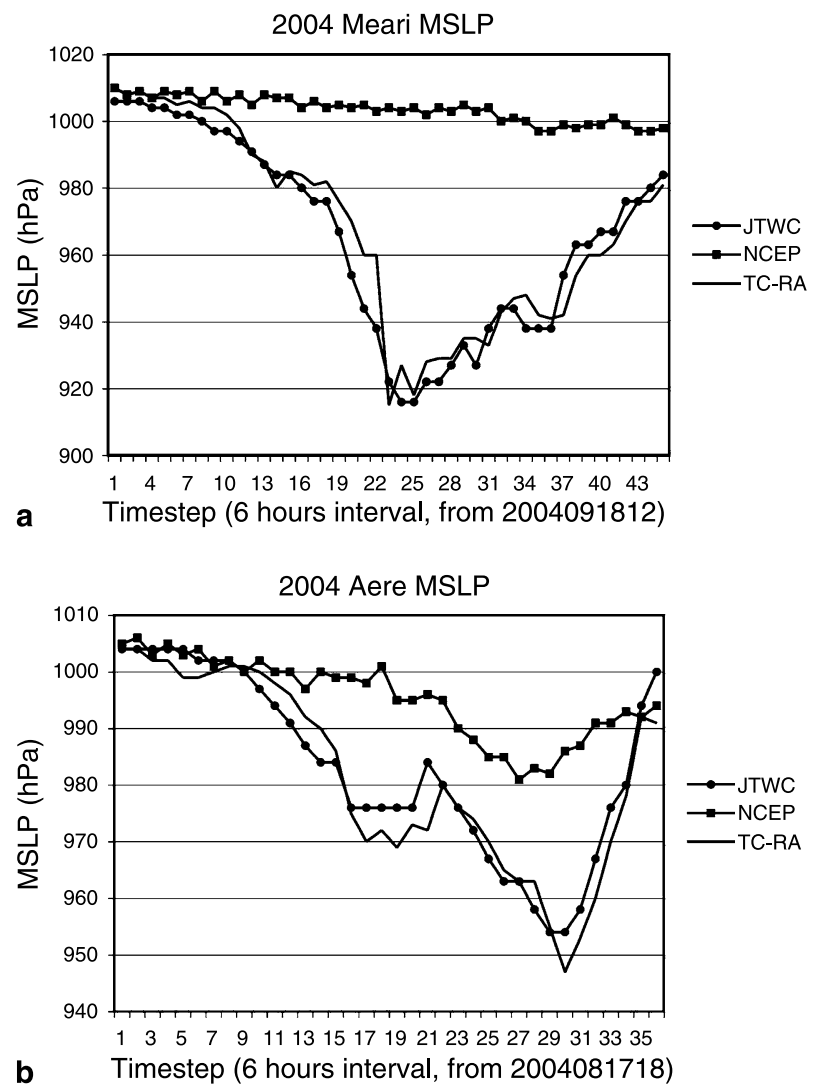

Fig. 5. Time evolution of the minimum central pressure of typhoons Meari (a) and Aere (b) of the JTWC best track analysis (JTWC), NCEP final analysis (NCEP), and the TC-RA (TC-RA) 
Meari (WP25) and Aere (WP20). Typhoon Meari experiences a rapid intensification from hour 96 to hour 144, with its minimum central pressure dropping from $980 \mathrm{hPa}$ to $916 \mathrm{hPa}$, an averaged rate of $32 \mathrm{hPa} /$ day. Typhoon Aere, on the other hand, experiences a double intensification period during hours 72-192. While the NCEP
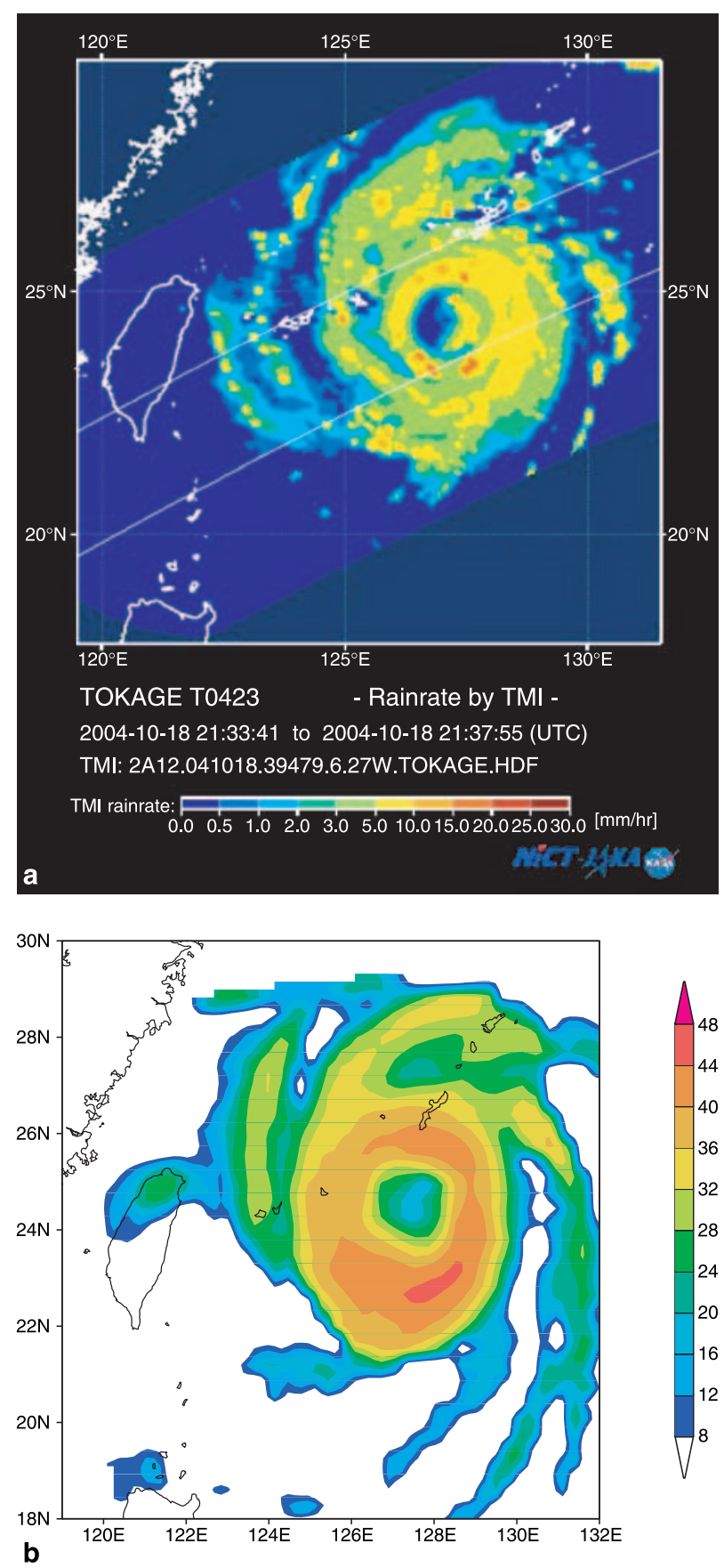

Fig. 6. Structure comparison for typhoon Tokage: (a) TMI rain rate observed at 2004-10-18 21:37:55 UTC, and (b) reanalyzed radar reflectivity at 2004-10-18 21:00:00 UTC final analysis failed to capture the rapid intensification for Meari and double intensification in Aere, the reanalysis product well captures their intensity changes. The results demonstrate the effectiveness of the combination of the 4DVAR assimilation system and the point-pressure scheme in successfully generating realistic TC intensity.
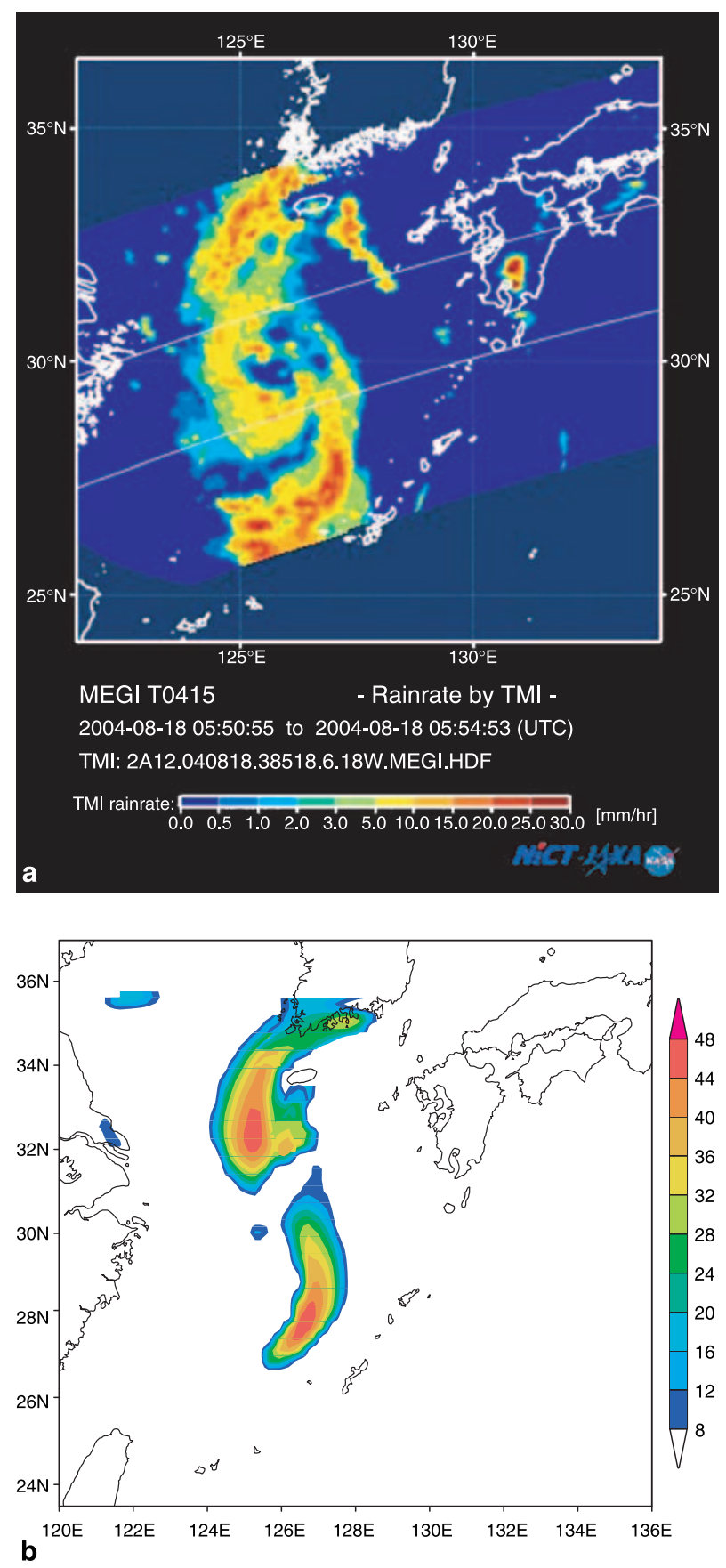

Fig. 7. Structure comparison for typhoon Megi: (a) TMI rain rate observed at 2004-08-18 05:54:53 UTC, and (b) reanalyzed radar reflectivity at 2004-08-18 06:00:00 UTC 


\subsection{TC rainband structure}

While the TC-RA product contains 3D dynamic and thermodynamic variables as well as hydrological parameters such as water vapor, cloudy water, rain water, ice, snow and graupel, our initial validation is focused on TC rainband structure. Below we present some preliminary comparisons between the TC-RA simulated radar reflectivity and remote-sensed images from independent satellite observations that are not used in the data assimilation. The satellite images are from TMI rain rate fields. The radar reflectivity was estimated from the model cloud water, rainwater, and other ice particles such as graupel. The method for calculation of the model reflectivity was the same as that in Liu et al (1997) and it was based on the $R-Z$ relation of Jorgenson and Wills (1982), and Fujiyoshi et al (1990).

In Fig. 6a, the rainband structure of typhoon Tokage at 200410182100 UTC was well captured by the TMI image, including a well-defined eyewall and outer convective rain bands. Along the eye wall, there is an asymmetry in the rain band, with the strongest convection occurring to the north and south of the TC center. The assimilated radar reflectivity near the same time showed a similar asymmetric inner-core structure, that is, the strongest reflectivity appeared in the north and south sections of the typhoon center (Fig. 6b). The shape of outer convective rain bands was asymmetric, with a strong branch from the north, consistent with the TRMM observation. Thus the reanalysis product reproduces the realistic intense inner and outer cloud/rain bands, in accordance with the satellite observations.

Figure 7 shows a similar comparison of the horizontal structure for typhoon Megi at 2004081806
UTC. At this time, a highly asymmetric spiral cloud band structure, with the highest rain rates over the northern and southern branches, appeared in the outer region (Fig. 7a). The TC-R A-assimilated radar reflectivity exhibits the bands of maximum intensity that extend from the typhoon center southward and northward, respectively (Fig. 7b). Thus the organized spiral cloud/rain bands pattern matches the main feature of the satelliteobserved rain band in TRMM TMI images.

The structure comparisons above demonstrate that the TC-RA product is able to reproduce the main character of TC rain bands and cloud reflectivity. This represents an encouraging improvement compared to the current operational analysis.

\subsection{Hindcast experiments}

One way to measure the quality of the TC-RA product is to conduct TC forecast experiments using the reanalysis product as an initial condition. By comparing the forecast results with and without the assimilated data, one may assess to what extent the TC forecast is improved due to the 4DVAR data assimilation. Here we present hindcast experiments for typhoon Tokage. Figure 8 shows the time evolution of the model simulated MSLP from October 14 to 19, with an initial condition from both the TC-RA and the NCEP global analysis. For validation, the observed temporal evolution of the MSLP from the JTWC best track is also plotted. The forecast with the TC-RA initial condition undergoes a rapid intensification, and the MSLP reaches as low as $920 \mathrm{hPa}$ after 72-hr integration. Although the forecast from the NCEP initial condition also

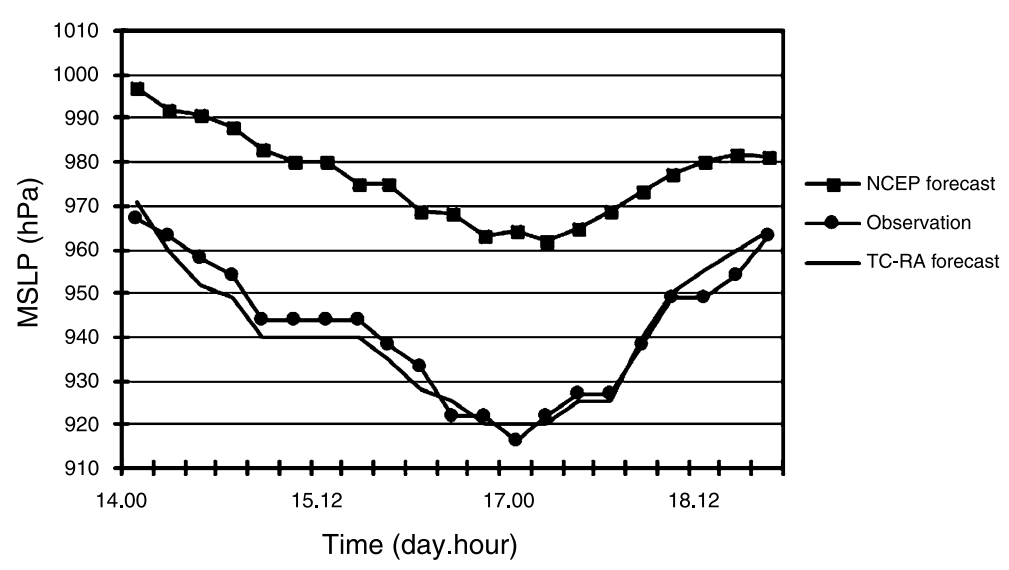

Fig. 8. Time series of the model forecasted Tokage MSLP from 2004101400Z to 2004101900Z, with an initial condition from the TC-RA and the NCEP global analysis. The solid line with dot marker is the observed MSLP from the JTWC best track 
intensifies, the maximum TC intensity remains above $960 \mathrm{hPa}$. The numerical results show a significant improvement in the TC intensity forecast when the model is initialized with the TC-RA product compared to that initialized with the NCEP global analysis. This suggests that the TC reanalysis is useful for not only producing better TC vortex structures but also improving subsequent intensity forecasts. In addition, the track forecast is also improved when using the TC-RA product, although it is not as significant as the intensity forecast (figure not shown).

\section{Conclusion and discussion}

A high-resolution, comprehensive TC reanalysis dataset is generated, with the application of the latest remotely sensed satellite observations and a 4DVAR assimilation technique. This reanalysis effort, supported by NOAA PRIDE program, is to construct a dataset that covers the entire life cycle of selected western Pacific typhoons in 2004. Up to now, 12 typhoons have been reanalyzed. The data assimilation system, the justification of each type of observational data in TC initialization and the preliminary verifications are presented. The main points are summarized as follows.

(1) An upgraded version of the MM5 adjoint system with a JcDFI penalty term and the corresponding minimization procedure successfully assimilates the best track information, GOES-derived wind, SSM/I rain rate, QuikSCAT surface wind, and Aqua retrieved temperature and water vapor fields into the MM5 model. This 4DVAR assimilation approach derives a dynamically consistent optimal initial condition for TCs. The minimization of the cost function in the current assimilation system is able to adjust the model initial condition in such a way that the model states are quite close to observations.

(2) The reanalysis product has much improved TC intensity and track compared to the NCEP global final analysis. The average track analysis error reduces from $60 \mathrm{~km}$ to $30 \mathrm{~km}$, while the intensity analysis bias improves from $30 \mathrm{hPa}$ to $5-10 \mathrm{hPa}$.

(3) A validation of the TC structure against independent satellite observations (radar reflectiv- ity vs. TMI images) shows that the reanalysis product reconstructs the main characteristics of asymmetric TC rain bands and spiral cloud bands.

(4) Numerical experiments show that TC intensity forecast is improved when the model is initialized with the TC-RA product compared to that initialized with the NCEP global analysis.

The results above point out that the assimilation of multi-satellite observations using 4DVAR has great potential for improving $\mathrm{TC}$ analysis and prediction. However, several problems pertinent to the reanalysis are worth noting for future improvement. First, the current $30-\mathrm{km}$ horizontal resolution is still coarse to resolve the inner core TC structures. This problem is mainly attributed to the huge amount of computational requirement of 4DVAR and the limitation of our computer resources. It is desired to increase the horizontal resolution to at least $10 \mathrm{~km}$. We anticipate that this increased resolution would lead to further improvement in the intensity and track reanalysis. The second problem is the cold-start during the assimilation cycle. This cold start may sometimes over-fit the vortex bogus data during the data assimilation. The third problem is the limited observational frequency from a single polar-orbiting satellite, which at most provides twice-daily regional coverage. The combined observations from multiple satellites such as those from NOAA 15 , 16, 18 and NASA Aqua and Terra may increase the temporal coverage of up to 10 times per day in a particular region. This certainly will improve the reanalysis quality.

We plan to further improve the quality of the reanalysis product in the near future and extend the reanalysis effort to cover western Pacific TCs in 2005-2006 and TCs in other ocean basins, with increased resolution $(<10 \mathrm{~km})$ and more remotely sensed observations. We plan to adopt a "warm-start" initialization strategy other than the "cold-start", so that the first guess comes from the previous assimilation 6 hours before instead of using the NCEP global analysis. This may improve the temporal consistency of the reanalysis product. In addition, we are plan to adopt NAVDAS of Naval Research Laboratory, which is a data assimilation system with the ability of handling more observational data and including 
basic ocean data analysis (Daley and Barker, 2001). Furthermore, we plan to adopt a socalled hybrid variational scheme (Weng et al, 2006), which directly assimilates microwave radiances in cloud- and rain-affected areas through a community radiative transfer model (CRTM) and then inputs the newly analyzed temperature products into the 4DVAR system. A significant improvement should be expected for the new product.

The TC reanalysis product will be posted on the data server of the Asia-Pacific Data-Research Center (http://apdrc.soest.hawaii.edu/) at the International Pacific Research Center. The current format of the reanalysis product is the MM5 model output format on a regular grid and $\sigma$ vertical levels, but a tool is available to convert the output format to the NetCDF format based on latitude-longitude grids and standard pressure levels. The output variables include 3D wind, temperature, water vapor, and hydrological parameters. Readers may refer to the MM5 documentation for a full list of the available variables.

\section{Acknowledgements}

This work was supported by the NOAA PRIDE project under grant NA17RJ1230. XZ is partial supported by China National Natural Sciences Foundation under grant 40305014. International Pacific Research Center is partially sponsored by the Japan Agency for Marine-Earth Science and Technology (JAMSTEC). This is SOEST contribution number 6803 and IPRC contribution number 434.

\section{References}

Bennett AF, Leslie LM, Gagelberg CR, Powers PE (1993) Tropical cyclone prediction using a barotropic model initialized by a generalized inverse method. Mon Wea Rev 121: 1714-1729

Bennett AF, Chua BS, Leslie LM (1996) Generalized inversion of a global numerical weather prediction model. Meteor Appl Phys 60: 165-178

Bourassa MA, Legler DM, O'Brien JJ, Smith SR (2003) SeaWinds validation with research vessels. J Geophys Res 108: 3019 (DOI:10.1029/2001JC001028)

Chen SS, Tenerelli JE, Zhao W, Foster RA, Liu WT (2004) Improving tropical cyclone prediction using scatterometer surface winds in model initialization. ENVSAT Symposium Abstract and Program Book, Salzburg, Austria, No. 669

Chen Y, Snyder C (2006) Assimilating vortex position with an ensemble Kalman filter. Mon Wea Rev (in revision)

Daley R, Barker E (2001) NAVDAS Source Book 2001. NRL Publication NRL/PU/7530-01-441, 163 pp
DeMaria M, Mainelli M, Shay LK, Knaff JA, Kaplan J (2005) Further improvement to the statistical hurricane intensity prediction scheme (SHIPS). Wea Forecast 20: $531-543$

Dudhia J (1993) A nonhydrostatic version of the Penn StateNCAR mesoscale model: validation tests and simulation of an Atlantic cyclone and cold front. Mon Wea Rev 121: 1493-1513

Ebuchi NH, Graber HC, Caruso MJ (2002) Evaluation of wind vectors observed by QuikSCAT/SeaWinds using ocean buoy data. J Atmos Oceanic Technol 19: 2049-2062

Freilich MH, Dunbar RS (1999) The accuracy of the NSCAT 1 vector winds: comparison with national data buoy center buoys. J Geophys Res 104: 11,231-11,246

Fujita T (1952) Pressure distribution within a typhoon. Geophys Mag 23: 437-451

Fujiyoshi Y, Endoh T, Yamada T, Tsuboki K, Tachibana Y, Wakahana G (1990) Determination of a Z-R relationship for snowfall using a radar and high sensitivity snow gauges. J Appl Meteor 29: 147-152

Gauthier P, Thépaut J-N (2001) Impact of the digital filter as a weak constraint in the preoperational 4DVAR assimilation system of Météo-France. Mon Wea Rev 129: 2089-2102

Grell GA, Dudhia J, Stauffer DR (1995) A description of the fifth-generation Penn State/NCAR Mesoscale Model (MM5). NCAR Tech. Note NCAR/TN-398+STR, 138 pp [Available from NCAR Publications Office, P.O. Box 3000, Boulder, CO 80307-3000]

Hayden CM, Purser RJ (1995) Recusive filter objective analysis of meteorological fields, applications to NESDIS operational processing. J Appl Meteor 34: 3-15

Holland GJ (1980) An analytic model of the wind and pressure profiles in hurricanes. Mon Wea Rev 108: 1212-1218

Hollinger JP (1991) DMSP Special Sensor Microwave/ Imager calibration/validation. Final Report, Vol. II, Naval Research Laboratory, Washington, DC, 106 pp [Available from Hollinger JP, Naval Research Laboratory, Washington, DC 20375]

Holmlund K (1998) The utilization of satellite properties of satellite-derived atmospheric motion vectors to derive quality indicators. Wea Forecast 13: 1093-1104

Holmlund K, Velden CS, Rohn M (2001) Enhanced automated quality control applied to high-density satellitederived winds. Mon Wea Rev 129: 517-529

Huang XY, Lynch P (1993) Diabatic digital-filtering initialization: Application to the HIRLAM model. Mon Wea Rev 121: 589-603

Isaksen L, Stoffelen A (2000) ERS scatterometer wind data impact on ECMWF's tropical cyclone forecasts. IEEE Trans Geosci Remote Sens 38: 1885-1892

Jorgenson DP, Wills PT (1982) A Z-R relationship for hurricanes. J Appl Meteor 21: 356-366

Kain JS, Fritsch JM (1990) A one-dimensional entraining/ detraining plume model and its application in convective parameterization. J Atmos Sci 47: 2784-2802

Kain JS, Fritsch JM (1993) Convective parameterization for mesoscale models: The Kain-Fritsch scheme. The representation of cumulus convection in numerical models. Meteor Monogr 24: 165-170 
Karyampudi VM, Lai GS, Manobianco J (1998) Impact of initial conditions, rainfall assimilation, and cumulus parameterization on simulations of hurricane Florence. Mon Wea Rev 126: 3077-3101

Kuo HL (1974) Further studies of the parameterizations of the influence of cumulus convection on large scale flow. J Atmos Sci 31: 1232-1240

Le Marshall JF, Smith W, Gallan G (1985) hurricane Debby - An illustration of the complementary nature of VAS soundings and cloud and water vapor motion winds. Bull Amer Meteor Soc 66: 258-263

Ledvina DV, Pfaendtner J (1995) Inclusion of Special Sensor Microwave/Imager (SSM/I) total precipitable water estimates into the GEOS-1 data assimilation system. Mon Wea Rev 123: 3003-3015

Leidner S, Isaksen L, Hoffman RN (2003) Impact of NSCAT winds on tropical cyclones in the ECMWF 4DVAR assimilation system. Mon Wea Rev 131: 3-26

Leslie LM, Le Marshall JF, Morison RP, Spinoso C, Purser RJ, Pescod N, Seecamp R (1998) Improved hurricane track forecasting from the continuous assimilation of high quality satellite wind data. Mon Wea Rev 126: $1248-1257$

Liu DC, Nocedal J (1989) On the limited memory BFGS method for large scale optimization. Math Program 45: $503-528$

Liu WT (2002) Progress in scatterometer application. J Oceanogr 58: 121-136

Liu Y, Zhang D-L, Yau MK (1997) A multiscale study of hurricane Andrew (1992). Part I: Explicit simulation and verification. Mon Wea Rev 125: 3073-3093

Lynch P, Huang XY (1992) Initialization of the HIRLAM model using a digital filter. Mon Wea Rev 120: 1019-1034

Mills GA, Gallan GM, Goodman BM (1986) A mesoscale data assimilation scheme for real time use in the McIDAS environment. CIMSS Tech. Report, 86 pp [Available from CIMSS, Space Science and Engineering Center, University of Wisconsin, 1225 West Dayton St., Madison, WI 53706]

Nieman SJ, Menzel WP, Hayden CM, Gray D, Wanzong S, Velden CS, Daniels J (1997) Fully automated cloud drift winds in NESDIS operations. Bull Amer Meteor Soc 78: 1121-1134

Park K, Zou X (2004) Toward developing an objective 4DVAR BDA scheme for hurricane initialization based on TPC observed parameters. Mon Wea Rev 132: 2054-2069

Peng MS, Chang SW (1996) Impacts of SSM/I-retrieved rainfall rates on numerical precipitation of a tropical cyclone. Mon Wea Rev 124: 1181-1198

Peng S-Q, Zou X (2002) Assimilation of NCEP multi-sensor hourly rainfall data using 4D-var approach: a case study of the squall line on April 5, 1999. Meteorol Atmos Phys 81: 237-255

Pickett MH, Tang W, Rosenfeld LK, Wash CH (2003) QuikSCAT satellite comparisons with near-shore buoy wind data off the US West Coast. J Atmos Oceanic Technol 20: 1869-1879

Price JC (1976) The nature of multiple solutions for surface wind speed over the oceans from scatterometer measurements. Remote Sens Environ 5: 47-54
Pu Z-X, Braun SA (2001) Evaluation of Bogus vortex techniques with four-dimensional variational data assimilation. Mon Wea Rev 129: 2023-2039

$\mathrm{Pu}$ Z-X, Wei-Kuo T, Scott B, Joanne S, Yiqin J, Jeffrey H, William O, Arthur H (2002) The impact of TRMM data on mesoscale numerical simulation of supertyphoon Paka. Mon Wea Rev 130: 2448-2458

$\mathrm{Pu}$ Z-X, Wei-Kuo Tao (2004) Mesoscale assimilation of TMI rainfall data with 4DVAR: Sensitivity studies. J Meteor Soc Japan 82: 1389-1397

Reisner J, Rasmussen RM, Bruintjes R (1998) Explicit forecasting of supercooled liquid water in winter storms using the MM5 model. Quart J Roy Met Soc 124: 1071-1107

Ruggiero FH, Michalakes J, Nehrkorn T, Modica GD, Zou X (2006) Development of a new distributed-memory MM5 adjoint. J Atmos Ocean Technol 23 (DOI: 10.1175/ JTECH1862.1, 424-436)

Shi JJ, Chang SW, Raman S (1996) Impact on assimilations of dropsonde data and SSM/I rain rates on numerical prediction of hurricane Florence (1988). Mon Wea Rev 124: $1435-1448$

Soden B, Velden CS, Tuleya R (2001) The impact of satellite winds on experimental GFDL hurricane model forecasts. Mon Wea Rev 129: 835-852

Troen I, Mahrt L (1986) A simple model of the atmospheric boundary layer: sensitivity to surface evaporation. Bound Layer Meteorol 37: 129-148

Tsuyuki T (1997) Variational data assimilation in the Tropics using precipitation data. Part III: Assimilation of SSM/I precipitation rates. Mon Wea Rev 125: 1447-1464

Velden C, Hayden C, Menzel W, Franklin JL, Lynch JS (1992) The impact of satellite-derived winds on numerical hurricane track forecasts. Wea Forecast 7: $107-118$

Velden C, Hayden C, Nieman S, Menzel W, Wanzong S, Goerss J (1997) Upper-tropospheric winds derived from geostationary satellite water vapor observations. Bull Amer Meteor Soc 78: 173-195

Velden C, Olander T, Wanzong S (1998) The impact of multispectral GOES-8 wind information on Atlantic tropical cyclone track forecasts in 1995: Part I: Dataset methodology, description, and case analysis. Mon Wea Rev 126: 1202-1218

Wee T-K, Kuo Y-H (2004) Impact of a digital filter as a weaker constraint in MM5 4DVAR: an observing system simulation experiment. Mon Wea Rev 132: 543-559

Weng F, Zhu T, Yan B (2006) Use of rain-affected radiances from microwave observations for hurricane vortex analysis. J Atmos Sci (submitted)

Xiao Q, Zou X, Wang B (2000a) On the initialization and prediction of a landfalling hurricane using a variational bogus data assimilation scheme. Mon Wea Rev 128: 2252-2269

Xiao Q, Zou X, Kuo Y-H (2000b) Incorporating the S SM/ I-derived precipitable water and rainfall rate into a numerical model: a case study for the ERICA IOP-4 cyclone. Mon Wea Rev 128: 87-108 
Xiao Q, Zou X, Pondeca M, Shapiro MA, Velden C (2002) Impact of GMS-5 and GOES-9 satellite-derived winds on the prediction of a NORPEX extratropical cyclone. Mon Wea Rev 130: 507-528

Zhang D-L, Anthes RA (1982) A high-resolution model of the planetary boundary layer-sensitivity tests and comparisons with SESAME-79 data. J Appl Meteor 21: 1594-1609

Zhang X, Wang B, Zhang X (2003) Four-dimensional variational assimilation using rainfall observation in heavy rain simulation. Prog Nat Sci 13: 1329-1333

Zhang X, Xiao Q, Fitzpatrick P (2006) The impact of multisatellite data on the initialization and simulation of hurricane Lili's (2002) Rapid weakening phase. Mon Wea Rev (accepted)

Zhao Y, Wang B, Ji Z, Liang X, Deng G, Zhang X (2005) Improved track forecasting of a typhoon reaching landfall from four-dimensional variational data assimilation of AMSU-A retrieved data. J Geophys Res 110, D14101 (DOI: 10.1029/2004JD005267)
Zou X, Kuo Y-H (1996) Rainfall assimilation through an optimal control of initial and boundary conditions in a limited-area mesoscale model. Mon Wea Rev 124: 2859-2882

Zou X, Xiao Q (2000) Studies on the initialization and simulation of a mature hurricane using a variational bogus data assimilation scheme. J Atmos Sci 57: 836-860

Zou X, Navon IM, Ledimet FX (1992) Incomplete observations and control of gravity waves in variational data assimilation. Tellus 44A: 273-296

Zupanski D, Mesinger F (1995) Four-dimensional variational assimilation of precipitation data. Mon Wea Rev 123: $1112-1127$

Zupanski M, Zupanski D (2002) Four-dimensional variational data assimilation for the blizzard of 2000. Mon Wea Rev 130: 1967-1988

Corresponding author's address: Xin Zhang, IPRC/ SOEST, University of Hawaii at Manoa, 1680 East West Road, POST Bldg 401, Honolulu, HI 96822, USA (E-mail: zhangxin@hawaii.edu) 